\title{
Kuratowski measure of noncompactness and integro- differential equations in Banach spaces
}

\author{
Mariam B Traoréa ${ }^{\text {, Ouaténi Diallo }}{ }^{\mathrm{a}}$, Mamadou Abdoul Diop ${ }^{\mathrm{b}, *}$ \\ ${ }^{a}$ Des Techniques et des Technologies de Bamako, Ecole Doctorale des Sciences et Technologies du Mali, Université des Sciences, B.P. \\ E2528, Bamako, Mali. \\ ${ }^{b}$ Département de Mathématiques, Université Gaston Berger de Saint-Louis, UFR SAT, B.P. 234, Saint-Louis, Sénégal.
}

\begin{abstract}
This paper focuses on the existence of mild solutions in Banach space for a first order semi-linear integro-differential equation. The results are achieved with the fixed-point theorem and Kuratowski measure of noncompactness. We conclude this study with an example to illustrate our findings.
\end{abstract}

Keywords: Integro-differential equation, mild solution, fixed point, Kuratowski measure of noncompactness, resolvent operator. 2020 MSC: 34G20, 37L05, 47J35, 65J08.

(C)2021 All rights reserved.

\section{Introduction}

Integro-differential equations appear in many fields of sciences such as physics, biology and other fields of applied mathematics. In the last years, this kind of equations have received considerable attention, see for example $[2,4,13,15,16]$ and references therein.

Many researchers used the measure of noncompactness to study the existence of mild solution for various forms of integral equations, differential equations, and integro-differential equations. For more details on this subject we refer the reader to Mouffak et al. [6], Rodkhina and Sadovskii [1], Alvarez [7], Benchohra and Rezoug [5], and references therein.

Motivated by all these papers, we derive sufficient conditions for the existence of solution of the following class of integro-differential equation

$$
\left\{\begin{array}{l}
\mathrm{dL}(\mathrm{t})=\left[\mathcal{O} \mathrm{L}(\mathrm{t})+\int_{0}^{\mathrm{t}} \Gamma(\mathrm{t}-\mathrm{s}) \mathrm{L}(\mathrm{s}) \mathrm{d} s+\mathrm{g}\left(\mathrm{t}, \mathrm{L}(\mathrm{t}), \int_{0}^{\mathrm{t}} \mathrm{h}(\mathrm{t}, \mathrm{s}, \mathrm{L}(\mathrm{s})) \mathrm{ds}\right)\right] \mathrm{dt}, \mathrm{t} \in \mathrm{I}=[0, \mathrm{~T}], \\
\mathrm{L}(0)=\mathrm{l}_{0},
\end{array}\right.
$$

where $(X,|\cdot|)$ is a real Banach space, $\mathcal{O}$ is the infinitesimal generator of a strongly continuous semigroup of bounded linear operators $(S(t))_{t \geqslant 0}$ on $X$ and $\Gamma(t)$ is a linear closed operator on $X$ with domain $\mathrm{D}(\mathcal{O}) \subset \mathrm{D}(\Gamma)$.

\footnotetext{
*Corresponding author

Email addresses: traoremariam34@yahoo.fr (Mariam B Traoré), ouateni@yahoo.fr (Ouaténi Diallo),

mamadou-abdoul. diop@ugb.edu.sn (Mamadou Abdoul Diop)
}

doi: $10.22436 /$ jnsa.014.02.06

Received: 2020-06-03 Revised: 2020-06-20 Accepted: 2020-07-21 
The function $h: I \times I \times X \rightarrow X$, with $s \leqslant t$ for $(s, t) \in I \times I$ is continuous in $X, g: I \times X \times X \rightarrow X$ is a Caratheodory function.

In our current paper, we will investigate the existence of solutions for the previously mentioned integrodifferential system since this problem still has not been considered in the literature. Our contributions are: a general class of integro-differential equation is considered in this work. Then, using functional analysis methods, a set of sufficient conditions is proposed to ensure the existence of mild solutions. The results are established through the use of the theory of the resolvent operator in the Grimmer sense. We use the fact that the operator-norm continuity of the resolvent operator is equivalent to that of the semigroup. This property allows us to drop the supposition that the operator semigroup is compact. This is an interesting result in itself and is of key practical importance. In fact in some applications, it is not easy to show the resolvent family associated to (2.1) is immediately norm continuous. Our paper expands the usefulness of integro-differential equations as literature shows only results of such equations in semigroup.

The paper is exhibited as follows. In Section 2, we remember some concepts and facts regarding Kuratowski measure of noncompatness and the theory of resolvent operator. Section 3 provides our main results. We will present an illustration in Section 4 to explain our key findings.

\section{Preliminaries}

Throughout this paper $(X,|\cdot|)$ is a Banach space and $C(I, X)$ denote the Banach space of continuous functions from $I$ into $X$ furnished by the usual supremum norm

$$
\|\mathrm{L}\|=\sup _{\mathrm{t} \in \mathrm{I}}|\mathrm{L}(\mathrm{t})|, \quad \forall \mathrm{L} \in \mathrm{C}(\mathrm{I}, \mathrm{X}) .
$$

Next to be able to reach existence of mild solutions for (1.1), we recall some details on partial integrodifferential equations and resolvent operators that will be used to establish the key results.

We look at the following Cauchy problem

$$
\left\{\begin{array}{l}
x^{\prime}(t)=\mathcal{O} x(t)+\int_{0}^{t} \Gamma(t-s) x(s) d s \text { for } t \geqslant 0, \\
x(0)=x_{0} \in X
\end{array}\right.
$$

where $X$ is a Banach space, $\mathcal{O}$ and $\Gamma(t)$ are closed linear operators on $X$ with domains $D(\mathcal{O}) \subset D(\Gamma)$. $Y$ represents the Banach space $\mathrm{D}(\mathcal{O})$ furnished with the graph norm defined by $|y|_{Y}:=|\mathcal{O} y|+|y|$ for $y \in Y$. We denote by $C([0,+\infty) ; Y)$, and $\mathcal{B}(Y, X)$, the space of all continuous functions from $[0,+\infty)$ into $Y$, the set of all bounded linear operators from $Y$ into $X$, respectively. If $X=Y$, we write $\mathcal{B}(X)$ instead of $\mathcal{B}(X, X)$.

Definition 2.1 ([12]). We call resolvent operator for the system (2.1), a bounded linear operator valued function $R(t) \in \mathcal{B}(X)$ for $t \geqslant 0$, having the following properties:

a) $R(0)=I$ (identity operator on $X$ ) and $\|R(t)\| \leqslant M e^{\beta t}$ for some constants $M$ and $\beta$;

b) for each $x \in X, R(t) x$ is strongly continuous for $t \geqslant 0$;

c) $R(t) \in \mathcal{B}(Y)$ for $t \geqslant 0$, for $x \in Y, R(\cdot) x \in C^{1}([0,+\infty), X) \cap C([0,+\infty), Y)$ and

$$
R^{\prime}(t) x=\mathcal{O} R(t) x+\int_{0}^{t} \Gamma(t-s) R(s) x d s=R(t) \mathcal{O} x+\int_{0}^{t} R(t-s) \Gamma(s) x d s \text { for } t \geqslant 0 .
$$

Example 2.1. Let $\mathrm{X}=\mathbb{R}, \mathrm{O} y=2 \mathrm{y}$ and $\Gamma(\mathrm{t}) \mathrm{y}=-2 \mathrm{y}$ in equation (2.1). Then in that case, we have

$$
R(t) x_{0}=e^{t}(\cos t+\sin t) x_{0} \text { and } T(t) x_{0}=e^{2 t} x_{0} .
$$

Remark 2.2. The above example shows that in general, the resolvent operator $R(t)_{t \geqslant 0}$ for equation (2.1) does not satisfy the semigroup law, namely,

$$
R(t+s) \neq R(t) R(s) \text { for some } t, s>0 .
$$


The existence of resolvent operator has been discussed in [12]. In what follows, we suppose the following assumptions.

(H1) $\mathcal{O}$ is the infinitesimal generator of strongly continuous semigroup $(S(t))_{t \geqslant 0}$ on $X$.

(H2) For all $t \geqslant 0, \Gamma(t)$ is closed operator from $D(\mathcal{O})$ to $X$ and $\Gamma(t) \in \mathcal{B}(Y, X)$. For any $y \in Y$, the map $t \longmapsto \Gamma(t) y$ is bounded, differentiable and the derivative $t \longmapsto \Gamma^{\prime}(t) y$ is bounded uniformly continuous on $\mathbb{R}^{+}$.

Theorem 2.3 ([12]). Assume that (H1)-(H2) hold. Then there exists a unique resolvent operator of the Cauchy problem (2.1).

We have the following theorem that establishes the equivalence between the operator-norm continuity of the $\mathrm{C}_{0}$-semigroup and the resolvent operator for integral equations.

Theorem 2.4 ([11, Theorem 6]). Let $\mathcal{O}$ be the infinitesimal generator of $a \mathrm{C}_{0}$-semigroup $(\mathrm{T}(\mathrm{t}))_{\mathrm{t} \geqslant 0}$ and let $(\Gamma(\mathrm{t}))_{\mathrm{t} \geqslant 0}$ satisfies (H2). Then the resolvent operator $(\mathrm{R}(\mathrm{t}))_{\mathrm{t} \geqslant 0}$ for equation(2.1) is operator-norm continuous (or continuous in the uniform operator topology) for $\mathrm{t}>0$ if and only if $(\mathrm{T}(\mathrm{t}))_{\mathrm{t} \geqslant 0}$ is operator-norm continuous for $\mathrm{t}>0$.

Next we introduce the Kuratowski measure of noncompactness.

Definition 2.5. Let B be a bounded subset of a Banach space X. The Kuratowski measure of noncompactness $v$ is defined by

$$
v(B)=\inf \{\epsilon>0: \text { B has a finite cover by sets of diameter smaller than } \epsilon\} .
$$

The Kuratowski measure of noncompactness satisfies the properties described by the following lemmas.

Lemma 2.6 ([3]). Let $\mathrm{X}$ be a Banach space and $\mathrm{B}, \mathrm{C} \subset \mathrm{X}$ be bounded, then the following properties are satisfied:

1. $v(\mathrm{~B})=0$ if only if $\mathrm{B}$ is relatively compact;

2. $v(\overline{\mathrm{B}})=v(\mathrm{~B}) ; \overline{\mathrm{B}}$ the closure of $\mathrm{B}$;

3. $v(\mathrm{C})<v(\mathrm{~B})$, when $\mathrm{C} \subset \mathrm{B}$;

4. $v(C+B)<v(C)+v(B)$, where $C+B=\{d \mid d=b+c ; b \in B, c \in C\}$;

5. $v(r B)=|r| v(B)$ for all $r \in \mathbb{R}$;

6. $v(\operatorname{Conv}(B))=v(B)$, where $\operatorname{Conv}(B)$ is the convex hull of $B$;

7. $v(C \cup B)=\max (v(C), v(B))$;

8. $v(C \cup\{x\})=v(C)$ for all $x \in X$.

Denote by $\eta^{\top}(y, \epsilon)$ the modulus of continuity of $y$ on the interval $[0, T]$, i.e.,

$$
\eta^{\top}(L, \epsilon)=\sup \{|L(t)-L(s)| ; t, s \in I,|t-s| \leqslant \epsilon\} .
$$

Further we define

$$
\eta^{\top}(B, \epsilon)=\sup \left\{\eta^{\top}(L, \epsilon), L \in B\right\}, \quad \eta_{0}^{\top}=\lim _{\epsilon \rightarrow 0} \eta^{\top}(B, \epsilon) .
$$

Lemma 2.7 ([8]). Let X be a Banach space, B be a bounded subset of X. Then there is a countable and bounded set $\mathrm{B}_{0} \subset \mathrm{B}$ such that

$$
v(B) \leqslant 2 v\left(B_{0}\right) .
$$

Lemma 2.8 ([14]). Let $X$ be a Banach space, $B=\left\{l_{n}\right\}_{n=0}^{\infty} \subset C(I, X)$ be a bounded and countable set. Then $v(B(t))$ is Lebesgue integrable on $\mathrm{I}$, and

$$
v\left(\left\{\int_{0}^{t} l_{n}(s) d s\right\}_{n=0}^{\infty}\right) \leqslant 2 \int_{0}^{t} v(B(s)) d s, t \in I .
$$


Lemma 2.9 ([18]). Let $w(t), u(t)$ and $v(t)$ be real valued nonnegative continuous function defined on $\mathbb{R}^{+}$, for which the inequality

$$
w(t) \leqslant w_{0}+\int_{0}^{t} u(s)\left[w(s)+\int_{0}^{s} v(\xi) w(\xi) d \xi\right] d s,
$$

is satisfied for all $\mathrm{t} \in \mathbb{R}^{+}$, where $w_{0}$ is a nonegative constant, then

$$
w(t) \leqslant w_{0}\left[1+\int_{0}^{t} u(s)\left[\exp \left(\int_{0}^{s}(u(\xi)+v(\xi)) d \xi\right)\right] d s\right]
$$

for all $\mathrm{t} \in \mathbb{R}^{+}$.

Definition 2.10 ([6]). Let $E$ be a Banach space. A continuous map $G: \Omega \subset E \rightarrow E$ is called condensing with respect to a measure of noncompactness $v$ (or $v$ - condensing) if for every bounded not relatively compact set $B \subset \Omega$, we have

$$
v(G(B))<v(B) .
$$

Theorem 2.11 ([9]). Let $\mathrm{E}$ be a Banach space, $\Omega$ be a bounded open subset of $\mathrm{E}$ and $0 \in \Omega$. Suppose that $\mathrm{F}: \bar{\Omega} \rightarrow \mathrm{E}$ is $v$-condensing and assume that $\mathrm{u} \neq \lambda \mathrm{F}(\mathrm{u})$ for $\mathrm{u} \in \partial \Omega$ and $\lambda \in(0,1)$ hold. Then $\mathrm{F}$ has a fixed point in $\bar{\Omega}$.

\section{Main result}

Definition 3.1. We call mild solution of the system (1.1), a function $L \in C(I, X)$ for which the following integral equation is satisfied

$$
L(t)=R(t) L_{0}+\int_{0}^{t} R(t-s) g\left(s, L(s), \int_{0}^{s} h(s, \xi, L(\xi)) d \xi\right) d s .
$$

In order to prove our results, we introduce the following assumptions.

(H3) There is a continuous function $u: I \rightarrow \mathbb{R}^{+}$, such that

$$
|g(t, x, y)| \leqslant u(t)(|x|+|y|) \text { for all } x, y \in X \text { and a.e. } t \in I .
$$

(H4) There are integrable functions $\rho_{i}(i=1,2): I \rightarrow \mathbb{R}^{+}$, such that:

$$
v\left(g\left(t, O_{1}, O_{2}\right)\right) \leqslant \rho_{1}(t) v\left(O_{1}\right)+\rho_{2}\left(t_{2}\right) v\left(O_{2}\right),
$$

for a.e. $t \in I$ and $\mathrm{O}_{1}, \mathrm{O}_{2} \subset X$.

(H5) There is a continuous function $v: \mathrm{I} \rightarrow \mathbb{R}^{+}$, such that:

$$
|h(t, s, x)| \leqslant v(t)|x|,
$$

for a.e. $(t, s) \in\{(t, s) \in I \times I ; s \leqslant t\}$ and $x \in X$.

(H6) There is a constant $h^{*}>0$, such that:

$$
v(h(t, s, D)) \leqslant h^{*} v(D),
$$

for a.e. $(t, s) \in J=\{(t, s) \in I \times I ; s \leqslant t\}$ and $D \in X$.

Theorem 3.2. Assume that equation (2.1) has a resolvent operator $(\mathrm{R}(\mathrm{t}))_{\mathrm{t} \geqslant 0}$ which is continuous in the operatornorm topology for $\mathrm{t}>0$ and hypotheses (H1)-(H6) hold. Then there exists at least one mild solution of (1.1). 
Proof. Let $\mathrm{Q}: \mathrm{C}(\mathrm{J}, \mathrm{X}) \rightarrow \mathrm{C}(\mathrm{J}, \mathrm{X})$, be a operator defined by

$$
(\mathrm{QL})(\mathrm{t})=\mathrm{R}(\mathrm{t}) \mathrm{L}_{0}+\int_{0}^{\mathrm{t}} \mathrm{R}(\mathrm{t}-\mathrm{s}) \mathrm{g}\left(\mathrm{s}, \mathrm{L}(\mathrm{s}), \int_{0}^{s} \mathrm{~h}(\mathrm{~s}, \xi, \mathrm{L}(\xi)) \mathrm{d} \xi\right) \mathrm{ds} .
$$

A mild solution of the system (1.1) is a fixed point of the operator Q. Let $\mathrm{P}$ be a positive constant. We consider the set $B_{P}=\{L \in C(J, X):\|L\|<P\}$. The proof will be splited into the following steps.

Step 1. $Q\left(B_{P}\right)$ is bounded for any bounded set $B_{P}$. For $L \in B_{P}$, and $t \in I$, we have

$$
\begin{aligned}
|\mathrm{QL}(\mathrm{t})| & \leqslant\|\mathrm{R}(\mathrm{t})\|\left|\mathrm{L}_{0}\right|+\int_{0}^{\mathrm{t}}\|\mathrm{R}(\mathrm{t}-\mathrm{s})\|\left|g\left(\mathrm{~s}, \mathrm{~L}(\mathrm{~s}), \int_{0}^{\mathrm{s}} \mathrm{h}(\mathrm{s}, \xi, \mathrm{L}(\xi)) \mathrm{d} \xi\right)\right| \mathrm{d} s \\
& \leqslant \mathrm{~N}\left|\mathrm{~L}_{0}\right|+\mathrm{N} \int_{0}^{\mathrm{t}} \mathrm{u}(\mathrm{s})\left(|\mathrm{L}(\mathrm{s})|+\int_{0}^{\mathrm{s}} v(\mathrm{~s})|\mathrm{L}(\xi)| \mathrm{d} \xi\right) \mathrm{d} s \leqslant \mathrm{~N}\left|\mathrm{~L}_{0}\right|+\mathrm{NPT}\|\mathrm{u}\|\left(1+\frac{\mathrm{T}}{2}\|v\|\right)<\infty .
\end{aligned}
$$

Thus $\mathrm{Q}\left(\mathrm{B}_{\mathrm{P}}\right)$ is bounded for all $\mathrm{L} \in \mathrm{B}_{\mathrm{P}}$.

Step 2. $Q$ is continuous. To see this, let $\left(l_{n}\right)_{n \in N}$ be a convergent sequence in $B_{P}$ such that $l_{n} \rightarrow l$. Then we have, for all $t \in I$

$$
\left|\left(Q l_{n}\right)(t)-(Q l)(t)\right| \leqslant N \int_{0}^{t}\left|g\left(s, l_{n}(s), \int_{0}^{s} h\left(s, \xi, l_{n}(\xi)\right) d \xi\right)-g\left(s, l(s), \int_{0}^{t} h(s, \xi, l(\xi)) d \xi\right)\right| d s .
$$

Since $h$ is continuous and $g$ is a Caratheodory function, using the Lebesgue dominated convergence theorem, we obtain

$$
\left\|\mathrm{Q} l_{n}-\mathrm{Ql}\right\| \rightarrow 0 \text { as } \mathrm{n} \rightarrow+\infty,
$$

which implies that $\mathrm{Q}$ is a continuous operator on $\mathrm{B}_{\mathrm{P}}$.

Step 3. $Q\left(B_{p}\right)$ is equicontinuous. Let $t_{1}, t_{2} \in I$ with $t_{1}<t_{2}$ and $L \in B_{P}$. We have:

$$
\begin{aligned}
\left|(Q L)\left(t_{2}\right)-(Q L)\left(t_{1}\right)\right| \leqslant & \left|\int_{0}^{t_{1}}\left(R\left(t_{2}-s\right)-R\left(t_{1}-s\right)\right) g\left(s, L(s), \int_{0}^{s} h(s, \xi, L(\xi)) d \xi\right) d s\right| \\
& +\left|\int_{t_{1}}^{t_{2}} R\left(t_{2}-s\right) g\left(s, L(s), \int_{0}^{s} h(s, \xi, L(\xi)) d \xi\right) d s\right| \\
\leqslant & \int_{0}^{t_{1}}\left\|R\left(t_{2}-s\right)-R\left(t_{1}-s\right)\right\|_{B(X)} u(s)\left(|L(s)|+\int_{0}^{s} v(\xi)|L(\xi)| d \xi\right) d s \\
& +N \int_{t_{1}}^{t_{2}} u(s)\left(|L(s)|+\int_{0}^{s} v(\xi)|L(\xi)| d \xi\right) d s .
\end{aligned}
$$

We obtain

$$
\begin{aligned}
\left|(Q L)\left(t_{2}\right)-(Q L)\left(t_{1}\right)\right| \leqslant & P \int_{0}^{t_{1}}\left\|R\left(t_{2}-s\right)-R\left(t_{1}-s\right)\right\|_{B(X)} u(s)\left(1+\int_{0}^{s} v(\xi) d \xi\right) d s \\
& +N P \int_{t_{1}}^{t_{2}} \sup _{t \in I}|u(t)|\left(1+\int_{0}^{s} \sup _{t \in I}|v(t)| d \xi\right) d s \\
\leqslant & P \int_{0}^{t_{1}}\left\|R\left(t_{2}-s\right)-R\left(t_{1}-s\right)\right\|_{B(X)} u(s)\left(1+\int_{0}^{s} v(\xi) d \xi\right) d s+N P\|u\| \int_{t_{1}}^{t_{2}}(1+s\|v\|) d s \\
\leqslant & P \int_{0}^{t_{1}}\left\|R\left(t_{2}-s\right)-R\left(t_{1}-s\right)\right\|_{B(X)} u(s)\left(1+\int_{0}^{s} v(\xi) d \xi\right) d s \\
& +N P\|u\|\left(\left(t_{2}-t_{1}\right)+\frac{1}{2}\|v\|\left(t_{2}-t_{1}\right)^{2}\right) .
\end{aligned}
$$

By Theorem 2.4, the right-hand side of the above inequality tends to zero as $t_{2}-t_{1} \rightarrow 0$. Consequently $\mathrm{Q}\left(\mathrm{B}_{\mathrm{P}}\right)$ is equicontinuous. 
Setp 4. We show that $\mathrm{Q}$ is condensing operator. Let $v^{*}(\mathrm{D})$ be the measure of noncompactness defined on the family of bounded subsets of the space $C(I, X)$ by

$$
v^{*}(D)=\eta_{0}^{\top}(D)+\sup _{t \in I} e^{-\xi \rho(t)} \bar{v}(D(t)),
$$

where

$$
\rho(t)=4 N \int_{0}^{t}\left(\rho_{1}(s)+2 h^{*} s \rho_{2}(s)\right) d s, \xi \geqslant 1, \quad \bar{v}(D(t))=\sup _{s \in[0, t]} v(D(s)) .
$$

For all $D \subset B_{P}, Q(D)$ is bounded. Therefore, a countable set $D_{0}=\left\{l_{n}\right\}_{n=1}^{\infty} \subset D$ exists by Lemma 2.7 so that

$$
v(Q(D)(t)) \leqslant 2 v\left(Q\left(D_{0}\right)(t)\right) \text { for each } t \in I \text {. }
$$

By the properties of $v$, hypotheses (H3), (H4), (H5), and Lemmas 2.7 and 2.8, we obtain

$$
\begin{aligned}
v\left(Q\left(D_{0}\right)(t)\right) & \leqslant v\left(\left\{\int_{0}^{t} R(t-s) g\left(s, l_{n}(s), \int_{0}^{s} h\left(s, \xi, l_{n}(\xi)\right) d \xi\right) d s\right\}_{n=0}^{\infty}\right) \\
& \leqslant 2 N \int_{0}^{t}\left\{v\left(g\left(s, l_{n}(s), \int_{0}^{s} h\left(s, \xi, l_{n}(\xi)\right) d \xi\right)\right)\right\}_{n=0}^{\infty} d s \\
& \leqslant 2 N \int_{0}^{t}\left(\rho_{1}(s)\left\{v\left(l_{n}(s)\right)\right\}_{n=0}^{\infty} d s+\rho_{2}(s)\left\{v\left(\int_{0}^{t} h\left(s, \xi, l_{n}(\xi)\right) d \xi\right)\right\}_{n=0}^{\infty}\right) d s \\
& \leqslant 2 N \int_{0}^{t}\left(\rho_{1}(s)\left\{v\left(l_{n}(s)\right)\right\}_{n=0}^{\infty}+2 h^{*} \rho_{2}(s)\left\{\int_{0}^{s} v\left(l_{n}(\xi)\right) d \xi\right\}_{n=0}^{\infty}\right) d s \\
& \leqslant 2 N \int_{0}^{t}\left(\rho_{1}(s) v\left(D_{0}(s)\right)+2 h^{*} \rho_{2} \int_{0}^{s} v\left(D_{0}(\xi)\right) d \xi\right) d s \\
& \leqslant 2 N \int_{0}^{t}\left(\rho_{1}(s) v\left(D_{0}(s)\right)+2 h^{*} \rho_{2}(s) s \sup _{\xi \in[0, s]} v\left(D_{0}(\xi)\right)\right) d s \\
& \leqslant 2 N \int_{0}^{t}\left(\rho_{1}(s) \sup _{s \in[0, t]} v\left(D_{0}(s)\right)+2 h^{*} \rho_{2}(s) s \quad \sup _{\xi \in[0, t]} v\left(D_{0}(\xi)\right)\right) d s \\
& \leqslant 2 N \int_{0}^{t}\left(\rho_{1}(s)+2 h^{*} s \rho_{2}(s)\right) \sup _{s \in[0, t]} v\left(D_{0}(s)\right) d s .
\end{aligned}
$$

From inequality (3.1), it follows that

$$
v(Q D(t)) \leqslant 4 N \int_{0}^{t}\left(\rho_{1}(s)+2 h^{*} \rho_{2}(s) s\right) \bar{v}(D(s)) d s .
$$

Therefore, we know

$$
v(\mathrm{QD}(\mathrm{t})) \leqslant 4 \mathrm{~N} \int_{0}^{\mathrm{t}}\left(\rho_{1}(\mathrm{~s})+2 \mathrm{~h}^{*} s \rho_{2}(\mathrm{~s})\right) e^{\xi \rho(s)} e^{-\xi \rho(s)} \bar{v}(\mathrm{D}(\mathrm{s})) \mathrm{ds}
$$

then

$$
e^{-\xi \rho(t)} v(Q D(t)) \leqslant \frac{1}{\xi} \sup _{t \in I} e^{-\xi \rho(t)} \bar{v}(D(t)), \quad \text { hence } \quad e^{-\xi \rho(t)} \sup _{t \in I} v(Q D(t)) \leqslant \frac{1}{\xi} \sup _{t \in I} e^{-\xi \rho(t)} \bar{v}(D(t)) .
$$

Since $e^{-\xi \rho(t)} \sup _{s \in[0, t]} v(Q D(s)) \leqslant e^{-\xi \rho(t)} \sup _{t \in I} v(Q D(t))$, we obtain

$$
e^{-\xi \rho(t)} \sup _{s \in[0, t]} v(Q D(s)) \leqslant \frac{1}{\xi} \sup _{t \in I} e^{-\xi \rho(t)} \bar{v}(D(t)) .
$$


Then

$$
e^{-\xi \rho(t)} \bar{v}(\mathrm{QD}(\mathrm{t})) \leqslant \frac{1}{\xi} \sup _{\mathrm{t} \in \mathrm{I}} e^{-\xi \rho(\mathrm{t})} \bar{v}(\mathrm{D}(\mathrm{t})) .
$$

From Step 3 and inequality (3.2), we obtain

$$
v^{*}(\mathrm{QD}) \leqslant v^{*}(\mathrm{D})
$$

Consequently, $\mathrm{Q}$ is condensing.

Step 5. We show that there is an open set $O \subseteq C(I, X)$ with $L \neq \beta Q(L)$, for $\beta \in(0,1)$ and $L \in \partial O$. Let $L \in C(I, X)$, suppose that $L=\beta Q(L)$ for $\beta \in(0,1)$. Then

$$
L(t)=\beta R(t) L_{0}+\beta \int_{0}^{t} R(t-s) g\left(s, L(\xi), \int_{0}^{s} h(s, \xi, L(\xi)) d \xi\right) d s .
$$

By (H1)-(H3) and (H5), for each $t \in I$, we have

$$
\begin{aligned}
|\mathrm{L}(\mathrm{t})| & \leqslant\|\mathrm{R}(\mathrm{t})\|_{\mathrm{B}(\mathrm{X})}\left|\mathrm{L}_{0}\right|+\int_{0}^{\mathrm{t}}\|\mathrm{R}(\mathrm{t}-\mathrm{s})\|_{\mathrm{B}(\mathrm{X})} \mathrm{u}(\mathrm{s})\left(|\mathrm{L}(\mathrm{s})|+\int_{0}^{\mathrm{s}} v(\xi)|\mathrm{L}(\xi)| \mathrm{d} \xi\right) \mathrm{d} s \\
& \leqslant \mathrm{~N}\left|\mathrm{~L}_{0}\right|+\mathrm{N} \int_{0}^{\mathrm{t}} \mathrm{u}(\mathrm{s})\left(|\mathrm{L}(\mathrm{s})|+\int_{0}^{s} v(\xi)|\mathrm{L}(\xi)| \mathrm{d} \xi\right) \mathrm{d} s .
\end{aligned}
$$

By Lemma 2.9, we get

$$
\begin{aligned}
|\mathrm{L}(\mathrm{t})| & \leqslant \mathrm{N}\left|\mathrm{L}_{0}\right|\left(1+\int_{0}^{\mathrm{t}} \mathrm{Nu}(\mathrm{s}) \exp \left(\int_{0}^{\mathrm{s}}(\mathrm{Nu}(\xi)+v(\xi)) \mathrm{d} \xi\right) \mathrm{d} s\right) \\
& \leqslant \mathrm{N}\left|\mathrm{L}_{0}\right|\left(1+\mathrm{N} \int_{0}^{\mathrm{t}} \sup _{\mathrm{t} \in \mathrm{I}}|\mathrm{u}(\mathrm{t})| \exp \left(\int_{0}^{s}\left(\mathrm{~N} \sup _{\mathrm{t} \in \mathrm{I}}|\mathrm{u}(\mathrm{t})|+\sup _{\mathrm{t} \in \mathrm{I}}|v(\mathrm{t})|\right) \mathrm{d} \xi\right)\right) \\
& \leqslant \mathrm{N}\left|\mathrm{L}_{0}\right|(1+\mathrm{TN}\|\mathrm{u}\| \exp (\mathrm{T}(\mathrm{N}\|\mathrm{u}\|+\|v\|)))=\tau .
\end{aligned}
$$

Thus, we get $\|\mathrm{L}\| \leqslant \tau$. We consider the set

$$
\mathrm{O}=\{\mathrm{L} \in \mathrm{C}(\mathrm{I}, \mathrm{X}):\|\mathrm{L}\|<\tau+1\}
$$

By the choice of $O$, there is no $L \in \partial O$ thus $L=\beta Q(L)$, for $\beta \in(0,1)$. Thus by Theorem 2.11, we conclude that $\mathrm{Q}$ has a fixed point that is a solution of the system (1.1).

\section{Example}

In order to illustrate the previous theoretical results, we consider the following semilinear integrodifferential equation

$$
\begin{aligned}
\frac{\partial}{\partial t} x(t, \xi)= & {\left[\frac{\partial^{2} \chi(t, \xi)}{\partial \xi^{2}}+\tilde{b} \frac{\partial x(t, \xi)}{\partial \xi}+\tilde{c} x(t, \xi)\right]+\int_{0}^{t} \Xi(t-s)\left[\frac{\partial^{2} x(s, \xi)}{\partial \xi^{2}}+\tilde{b} \frac{\partial x(s, \xi)}{\partial \xi}+\tilde{c} x(s, \xi)\right] d s } \\
& +\frac{x(t, \xi)}{(\sqrt{t}+1)(1+|x(t, \xi)|)} \\
& +\frac{e^{-t}}{(\sqrt{t}+1)(t+1)} \int_{0}^{s} \frac{\sqrt{t} x(s, \xi)}{\left(1+s^{2}+t\right)\left(1+x^{2}(s, \xi)\right)} d s, t \in[0, T], \xi \in[0,1], \\
x(t, 0)= & x(t, 1)=0, \text { for } t \in[0, T], \\
x(0, \xi)= & x_{0}, \quad t \in[0, T], \xi \in[0,1],
\end{aligned}
$$


where $\Xi: \mathbb{R}^{+} \mapsto \mathbb{R}$ is continuous function, $\tilde{b}, \tilde{c} \in \mathbb{R}$.

Let $X=\mathrm{L}^{2}(0,1)$. We define the operator $\mathcal{O}$ induced on $X$ as follows:

$$
\left\{\begin{array}{l}
\mathrm{D}(\mathcal{O})=\mathrm{H}^{2}(0,1) \cap \mathrm{H}_{0}^{1}(0,1) \\
\mathcal{O} z=z^{\prime \prime}+\tilde{\mathrm{b}} z^{\prime}+\tilde{\mathrm{c}} z, \quad \tilde{\mathrm{b}}, \tilde{\mathrm{c}} \in \mathbb{R} .
\end{array}\right.
$$

From [10, p. 173], we know that $\mathcal{O}$ is the infinitesimal generator of an analytic $\mathrm{C}_{0}$ semigroup $(S(t))_{t \geqslant 0}$ on $X$. Since the semigroup generated by $\mathcal{O}$ is analytic, then it is norm continuous for $t>0$.

We define the operators $\Gamma(t): Y \mapsto X$ as follows:

$$
\Gamma(t) N=\Xi(t) \mathcal{O N} \text { for } t \geqslant 0 \text { and } N \in D(\mathcal{O}) .
$$

Furthermore, for every $t \in[0, T]$, we define

$$
\begin{aligned}
\mathrm{L}(\mathrm{t}) & =x(t, \xi), \\
g(t, y, z)(\xi) & =\frac{1}{(\sqrt{t}+1)(1+|y(t, \xi)|)} y(t, \xi)+\frac{e^{-t}}{(\sqrt{t}+1)(t+1)} z(t, \xi), \\
h(t, s, y)(\xi) & =\frac{\sqrt{t} y(t, \xi)}{\left(1+s^{2}+t\right)\left(1+y^{2}(t, \xi)\right)} .
\end{aligned}
$$

Thus, (4.1) takes the following abstract form

$$
\left\{\begin{array}{l}
\mathrm{dL}(\mathrm{t})=\left[\mathcal{O L}(\mathrm{t})+\int_{0}^{\mathrm{t}} \Gamma(\mathrm{t}-\mathrm{s}) \mathrm{L}(\mathrm{s}) \mathrm{d} s+\mathrm{g}\left(\mathrm{t}, \mathrm{L}(\mathrm{t}), \int_{0}^{\mathrm{t}} \mathrm{h}(\mathrm{t}, \mathrm{s}, \mathrm{L}(\mathrm{s})) \mathrm{ds}\right] \mathrm{dt}, \quad \mathrm{t} \in \mathrm{I}=[0, \mathrm{~T}],\right. \\
\mathrm{L}(0)=\mathrm{l}_{0} .
\end{array}\right.
$$

We suppose $\Xi$ is a bounded and $C^{1}$ function such that $\Xi^{\prime}$ is bounded and uniformly continuous which implies that the operator $\Gamma(t)$ satisfies (H2). Thus from Theorems 2.3 and 2.4, problem (4.2) has a resolvent operator $(R(t))_{t \geqslant 0}$ on $X$ which is norm continuous for $t>0$.

We have

$$
|g(t, y, z)| \leqslant \frac{1}{1+\sqrt{t}}(|y(t, \xi)|+|z(t, \xi)|),
$$

and

$$
|h(t, s, y)| \leqslant \frac{\sqrt{t}}{1+t}|y(t, \xi)| .
$$

Hence conditions (H3) and (H5) are satisfied with

$$
u(t)=\frac{1}{1+\sqrt{t}}, v(t)=\frac{\sqrt{t}}{1+t} .
$$

From the definition of $g$, for every $t \in I$, and $B_{1}, B_{2} \in B \subset X$, we have

$$
v\left(g\left(t, B_{1}, B_{2}\right)\right) \leqslant \frac{1}{\sqrt{t}+1} v\left(B_{1}\right)+\frac{e^{-t}}{(1+\sqrt{t})(1+t)} v\left(B_{2}\right) .
$$

Hence condition (H4) is satisfied with

$$
\rho_{1}(t)=\frac{1}{\sqrt{t}+1} \text { and } \rho_{2}=\frac{e^{-t}}{(1+\sqrt{t})(1+t)} .
$$

By (4.3), for every $t \in I$ and $B \subset X$, we have $v(h(t, s, B)) \leqslant \sup _{t \in I} \frac{\sqrt{t}}{t+1} v(B)$, then

$$
v(h(t, s, B)) \leqslant \frac{\sqrt{2}}{3} v(B) .
$$

Hence (H6) is satisfied with $h^{*}=\frac{\sqrt{3}}{2}$. Finally, all assumptions of our main results are satisfied. From Theorem 3.2, we deduce the existence of solution of system (4.1). 


\section{Conclusion}

This paper has studied a new class of partial integro-differential equations. The results are achieved with fixed-point theorem and Kuratowski measure of noncompactness. Finally, an example is given to illustrate the effectiveness of the results obtained. There is one direct issue which require further study. We will investigate the partial functional integro-differential with state-dependent nonlocal conditions by using a generalization of the classical Darbo fixed point theorem for Fréchet spaces associated with the concept of measures of noncompactness.

\section{Acknowledgment}

The authors would like to thank the anonymous referee for his/her carefully reading the manuscript and very important comments and suggestions that improved the results and quality of this paper.

\section{References}

[1] R. R. Akhmerov, M. I. Kamenskii, A. S. Patapov, A. E. Rodkhina, A. S. Rodkina, B. N. Sadovskii, Measures of noncompactness and condensing operators, Birkäuser Verlag, Basel, (1992). 1

[2] K. Balachandran, D. G. Park, S. M. Anthoni, Existence of solutions of abstract nonlinear second-order neutral functional integrodifferential equations, Comput. Math. Appl., 46 (2003), 1313-1324. 1

[3] J. Banas, K. Goebel, Measures of Noncompactness in Banach Spaces, Marcel Dekker, New York, (1980). 2.6

[4] A. Belleni-Morante, An Integrodifferential equation arising from the theory of heat conduction in rigid material with memory, Boll. Un. Mat. Ital., 15 (1978), 470-482. 1

[5] M. Benchohra, N. Rezzoug, Measure of noncompactness and second order evolution equations, Gulf J. Math., 4 (2016), 71-79. 1

[6] M. Benchohra, N. Rezoug, Y. Zhou, Semilinear Mixed Type Integro-Differential Evolution Equations Via Kuratowski Measure of Noncompactness, Z. Anal. Anwend., 38 (2019), 143-156. 1, 2.10

[7] J. Carmona Alvárez, Measure of Noncompactness and fixed points of nonexpansive condensing mapping in locally convex spaces, Rev. Real Acad. Cienc. Exact. Fís. Natur. Madrid, 79 (1985), 53-66. 1

[8] P. Y. Chen, Y. X. Li, Monotone Iterative Technique for a Class of Semilinear Evolution Equations with Nonlocal Conditions, Results Math., 63 (2013), 731-744. 2.7

[9] K. Deimling, Nonlinear Functional Analysis, Springer-Verlag, Berlin, (1985). 2.11

[10] K.-J. Engel, R. Nagel, One-parameter semigroups for linear evolution equations, Springer-Verlag, New York, (2000). 4

[11] K. Ezzinbi, G. Degla, P. Ndambomve, Controllability for some Partial Functional Integrodifferential Equations with Nonlocal Conditions in Banach Spaces, Discuss. Math. Differ. Incl. Control Optim., 35 (2015), 25-46. 2.4

[12] R. C. Grimmer, Resolvent operators for integral equations in a Banach space, Trans. Amer. Math. Soc., 273 (1982), $333-349$. $2.1,2.1,2.3$

[13] D. Guo, V. Lakshmikantham, X. Z. Liu, Nonlinear Integral Equation in Abstract Spaces, Kluwer Academic Publishers Group, Dordrecht, (1996). 1

[14] H. P. Heinz, On the behaviour of measure of noncompactness with respect to differentiation on integration of rector-valued functions, Nonlinear Anal., 7 (1983), 1351-1371. 2.8

[15] H. R. Henriquez, V. Poblete, J. C. Pozo, Mild solutions of non-autonomous second order problems with nonlocal initial conditions, J. Math. Anal. Appl., 412 (2014), 1064-1083. 1

[16] L. Olszowy, Existence of mild solutions for semilinear nonlocal Cauchy problems in separable Banach spaces, Z. Anal. Anwend., 32 (2013), 215-232. 1

[17] L. Olszowy, S. Wedrychowicz, On the existence and asymptotic behaviour of solutions of an evolution equation and an application to the Feynman-Kac theorem, Nonlinear Anal., 74 (2011), 6758-6769.

[18] B. G. Pachpatte, A note on Gronwall-Bellman inequality, J. Math. Anal. Appl., 44 (1973), 758-762. 2.9 